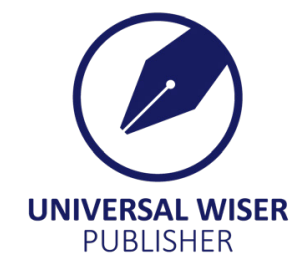

\title{
Blast Resistant Structure Using Steel Hollow Sections
}

\author{
A Saha Chaudhuri", D Sarkar \\ Civil Engineering Department, Techno Main Saltlake, Kolkata, India \\ Email: arupsc@rediffmail.com
}

\begin{abstract}
The number of terrorist attacks increased in the last few years, so the effect of blast loads on structures become an important matter which should be taken into consideration in the design and planning process. Terrorist attacks are exceptional cases and man-made disasters, so blast loads are calculated carefully just like earthquake and wind loads. The objective of this research is to determine the quality of blast load simulation for non-linear dynamic response analysis of trussed structure subjected to blast loads. Blast resistant structure design theories, the enhancement of building security against the effects of explosives in both architectural and structural design process are discussed in this paper. Steel hollow sections are used as structural members those can undergo large nonlinear deformations to absorb blast energy. Essential techniques for increasing the capacity of a structure to provide protection against blast effects are discussed in this paper both with an architectural and structural approach.
\end{abstract}

Keywords: blast load, non-linear dynamic analysis, blast resistant structure

\section{Introduction}

The terrorist activities and threats have become a growing problem all over the world and protection against terrorist acts involves prediction, prevention and mitigation of such events. Recent blast incidents in the world trigger the minds of developers, architects and engineers to find solutions to protect the buildings from blast disasters. Extensive research into blast effects analysis and techniques to protect buildings has been initiated in many countries to develop methods of protecting buildings and infrastructures. Blast loads are basically dynamic loads like wind and seismic loads. Blast loads are extreme, instantaneous, unpredictable impulses acting over milliseconds.

In this paper, a dome shape Blast Resistant Structure is designed and analysed using Non-Linear Dynamic Analysis in ETABS 2015. The structure is designed as a cover of an important building. The Blast Resistant Structure improves the building security against the effects of explosion. This is the interesting one in military circles and important data derived from the experience and tests have been restricted to army use. For structural members steel square hollow sections are used those can undergo large nonlinear deformations to absorb blast energy. Also dome shape is very strong against any kind of collapse of structures. The string vibration effects of structural members are observed against the blast loading that is applied instantaneously. Steel has sufficient elasto-plastic properties to take care of this load. ${ }^{[1],[5],[6]}$

\section{Explosion and Blast Phenomena}

The explosion is a phenomenon of rapid and abrupt release of energy. Explosive blast is quite different from other types of severe loads resulting from extreme events such as earthquake, impact or high wind. Blast loading is the result of an explosion where this refers to a rapid and sudden release of stored energy. Blast loads are applied extremely rapid and may last within a fraction of second. Explosive blast activates many structural response mechanisms because of its extreme spatial and time variations in magnitude and duration. When explosion takes place, the explosive materials disintegrate emitting the heat and producing gas.

The violent release of energy from a detonation in a gaseous medium gives rise to sudden pressure increase in that medium. The consequential pressure disturbance, termed as blast wave. Only one third of the total chemical energy is released by detonation. The remaining two thirds are released slowly in the blasts as the explosive materials mix with the surrounding air and burn.

The typical pressure profile of the blast wave in time for the explosion in the air is given in the following graph. ${ }^{[2],[3]}$ 


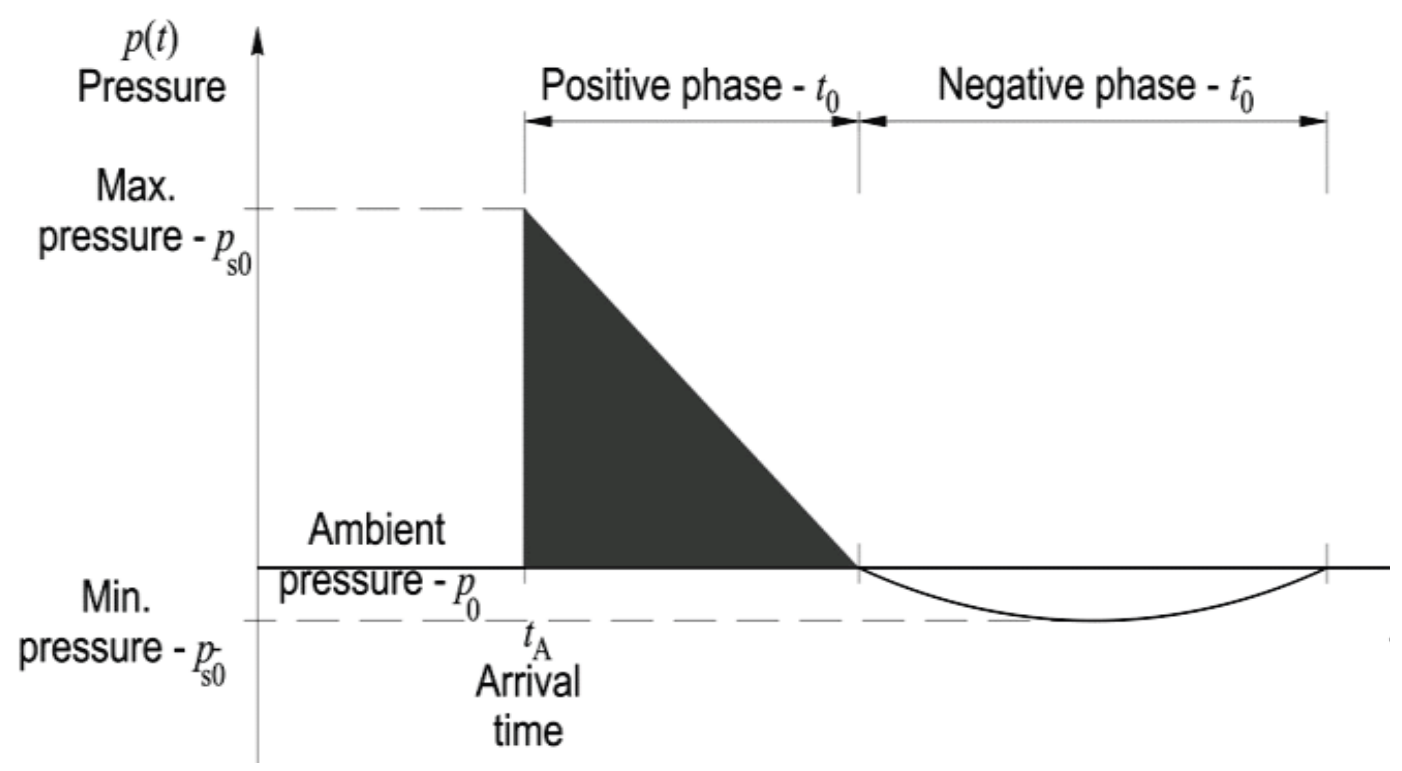

Figure 1. Pressure-Time Profile of the Blast Wave

$\mathrm{P}_{\mathrm{S} 0}=$ Peak static wave front overpressure.

$\mathrm{P}_{0}=$ Ambient pressure (atmospheric pressure).

$\mathrm{P}^{-}{ }_{\mathrm{S} 0}=$ Maximum value of negative pressure (pressure below ambient pressure) in the negative phase of the blast.

$\mathrm{t}_{0}=$ Duration of the positive phase during which the pressure is greater than the pressure of the surrounding air.

$\mathrm{t}_{0}^{-}=$Duration of the negative phase.

$\mathrm{t}_{\mathrm{A}}=$ Moment of arrival (Arrival time).

\section{Case Study}

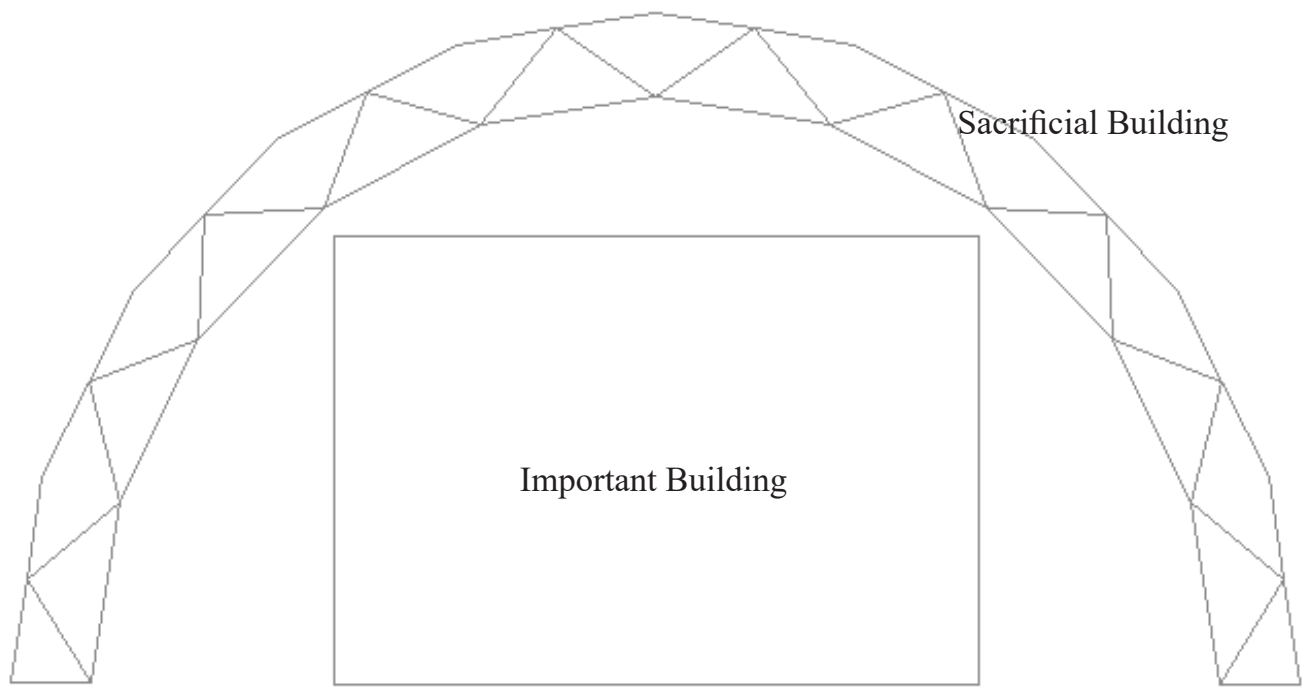

Figure 2. Front View of Internal RCC Building with Outside Protection of Steel Dome

A Blast Resistant Structure of dome shape is designed and analysed in ETABS 2015 by performing a Non-Linear Dynamic Analysis. The structure is a cover of an important building. The structure has a length of $24 \mathrm{~m}$ and height of $12 \mathrm{~m}$.

The structure is a steel framed structure, made with square hollow sections. Property of Tata Structura (Square Hollow 
Section) - SHS BxB $=132 \times 132 \mathrm{~mm}$ and Thickness $=5.40 \mathrm{~mm}$, is applied to the structure. Steel sheathing of thickness 3.4 $\mathrm{mm}$ (according to the manufacturer's specification) is provided on the structure. Pinned support is given to the base nodes of the structure. ${ }^{[4],[7]}$

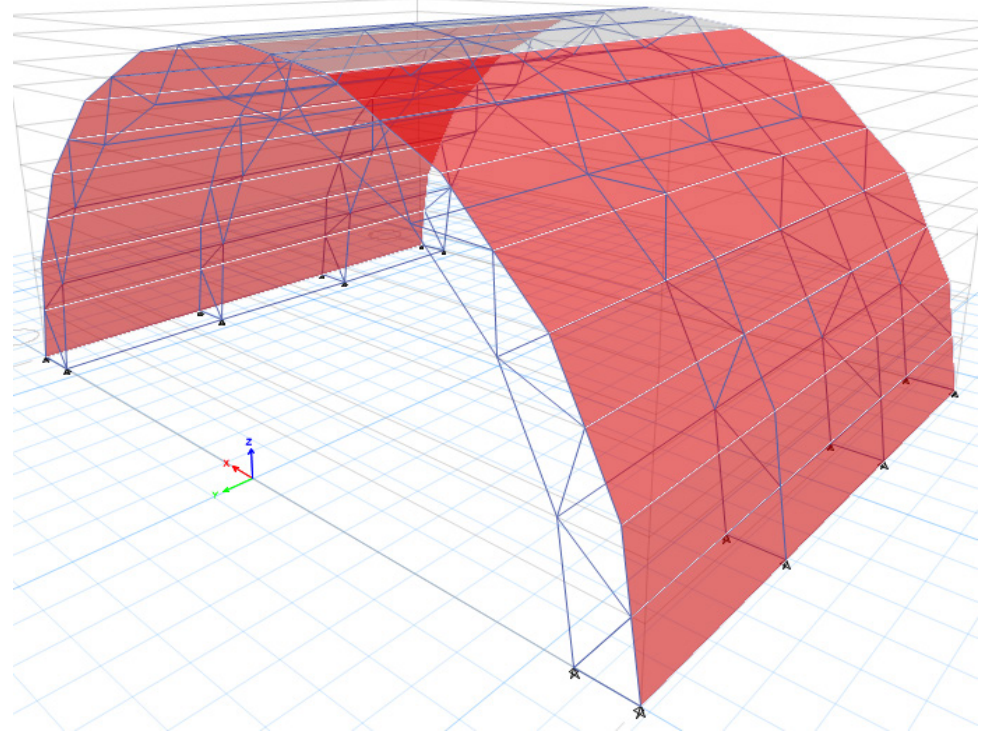

Figure 3. 3D View of Input Model

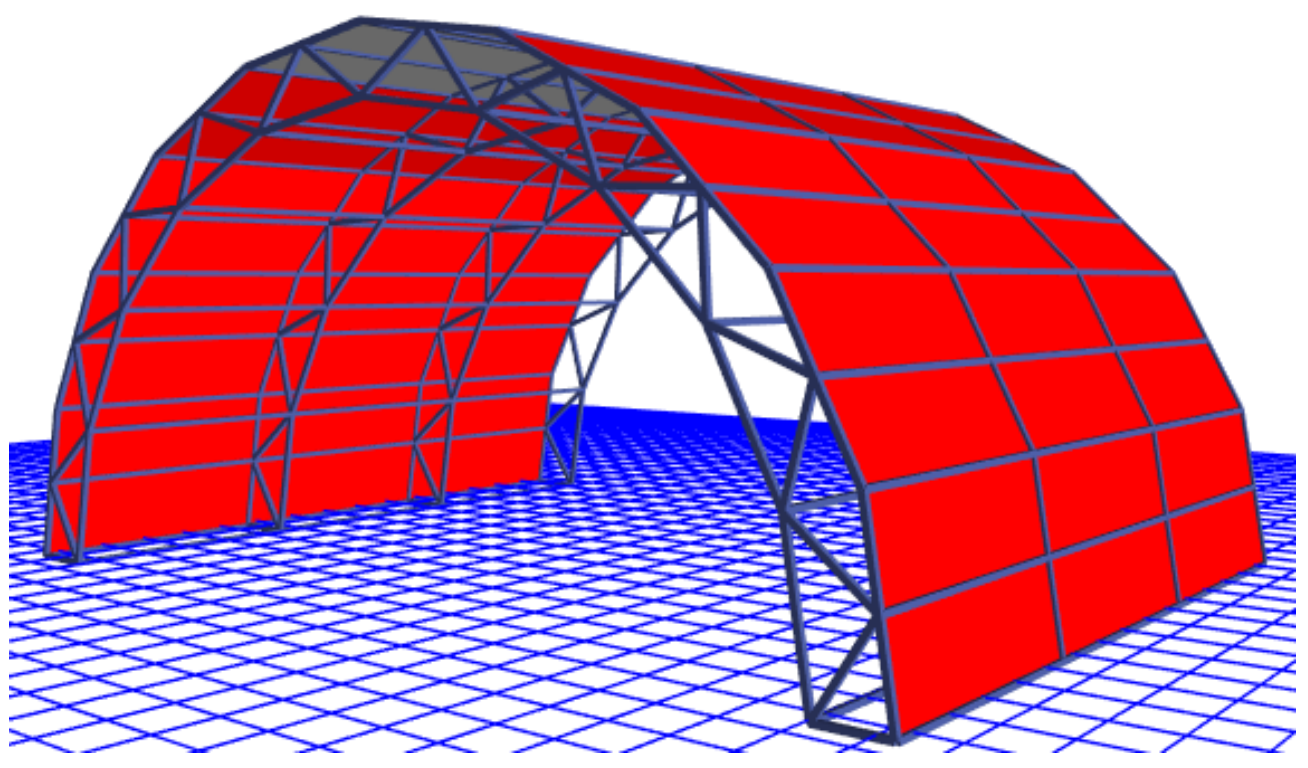

Figure 4. 3D Rendered View of Input Model

Dead load, live load, wind load as UDL and Nodal load $(18 \mathrm{KN})$ are applied on the structure. Nodal load $(18 \mathrm{KN})$ is given as peak blast load on the structure. The loads are given symmetrically.

Blast analysis can be carried out in ETABS 2015 by performing a time history analysis in which the blast loading is applied, typically using a triangular time function. The behaviour of the structure under the effect of blast loading can be studied from the output created by the ETABS 2015. The general approach for solving the dynamic response of blast resistant structure is Non-Linear Modal Analysis (FNA).

When blast load is applied on the structure, the structure absorbs the energy and vibrates like strings. After that the structure is deformed by releasing the energy. For that reason, the internal building is becoming safe after the blast. 


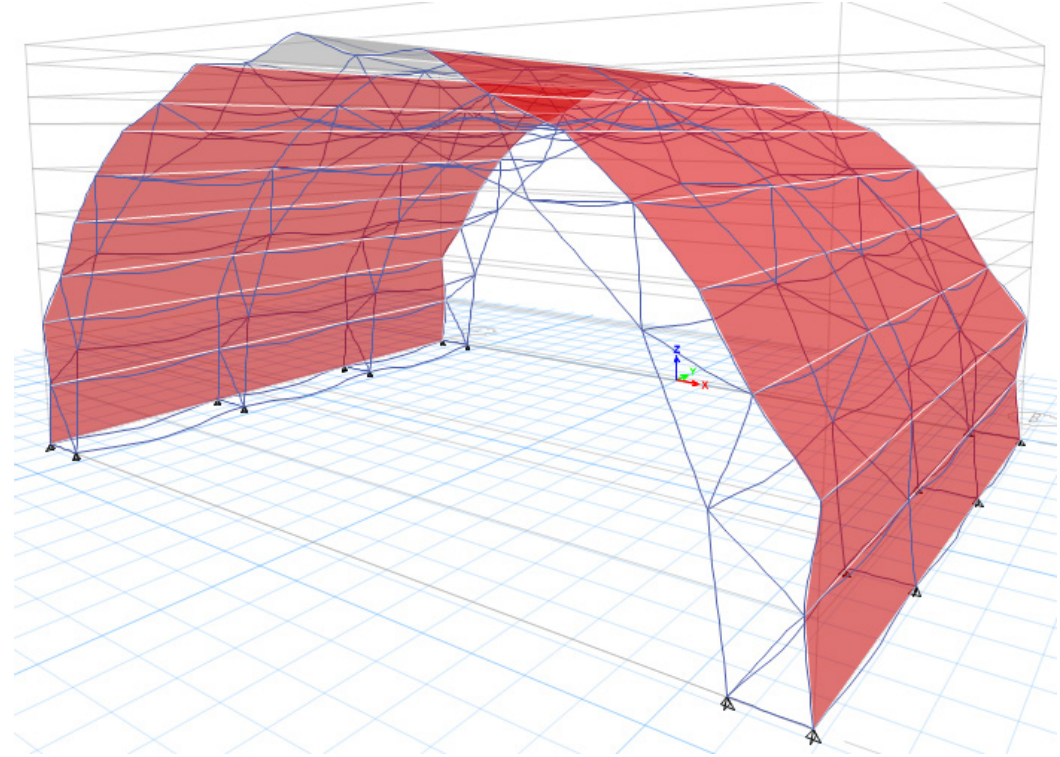

Figure 5. Deformed Shape of Output Model

\section{Results and Discussion}

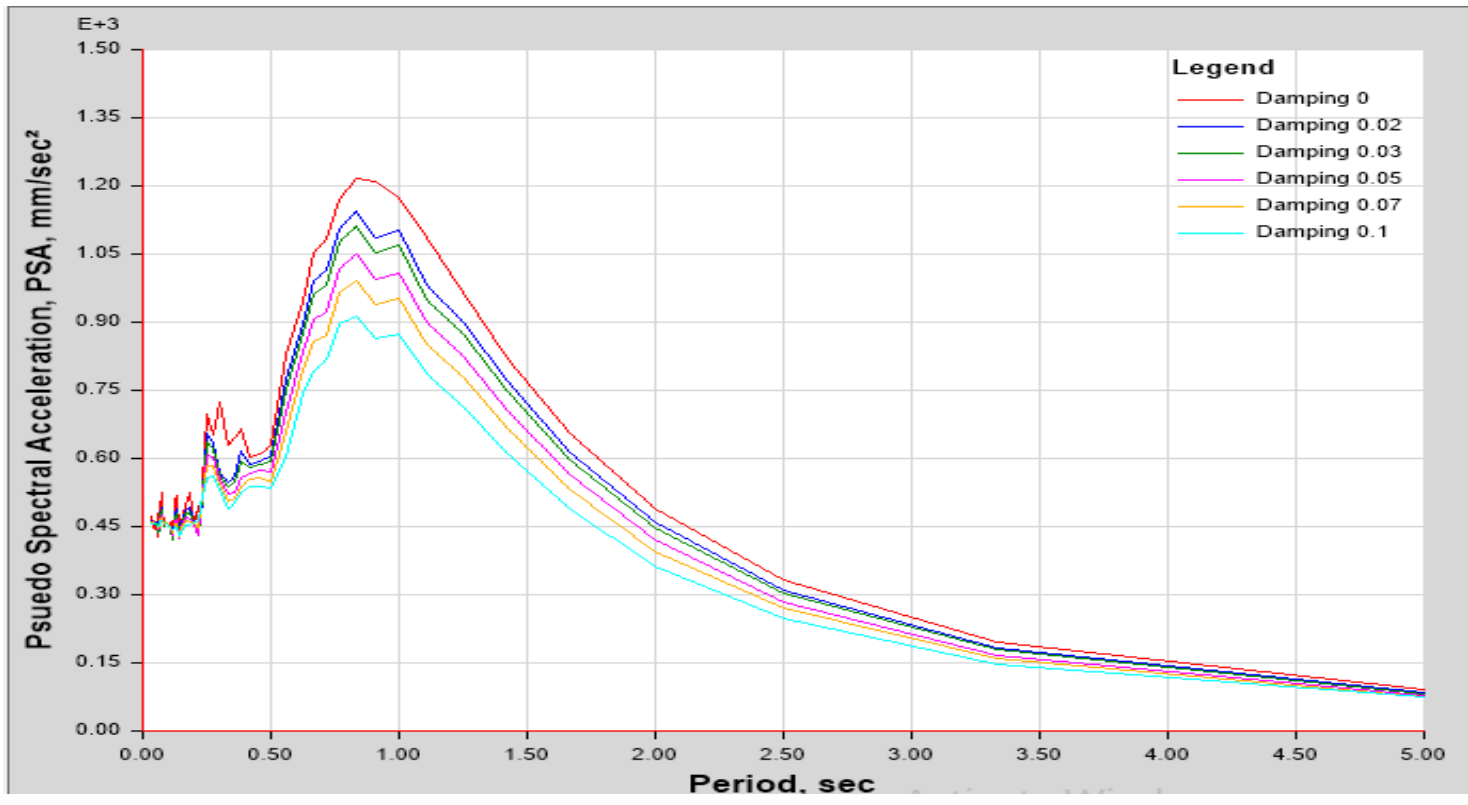

Figure 6. Response Spectrum Curve 


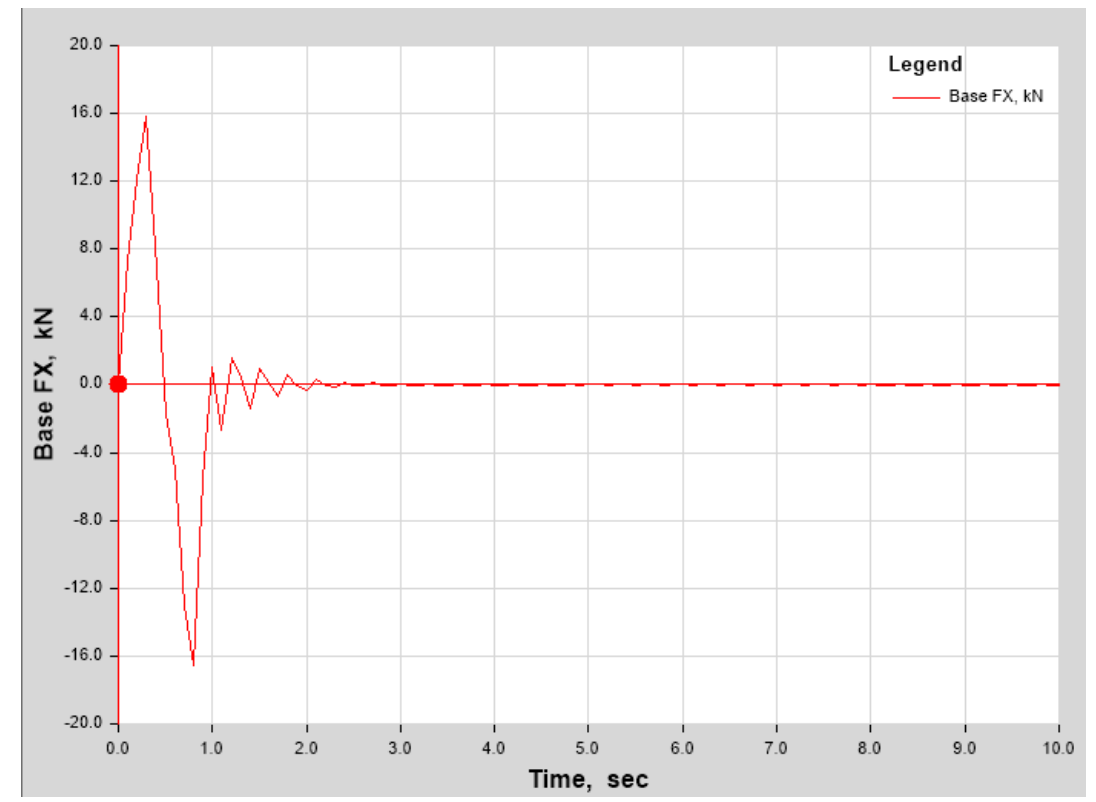

Figure 7. Base Shear Curve

From Figure 6 it is observed that initially the string vibration acceleration is very high but with the time it diminishes by releasing the energy through work done by the steel members.

From graphical representation of Time (sec) versus Base Shear FX (KN) it is evident that as blast load is applied on the structure, the structure vibrates for 2.5 seconds and then the structure is deformed by releasing energy.

Thus the vibration is generated and diminished in the cover structure only. In this study the string like vibration behaviour of slender members are observed and considered only.

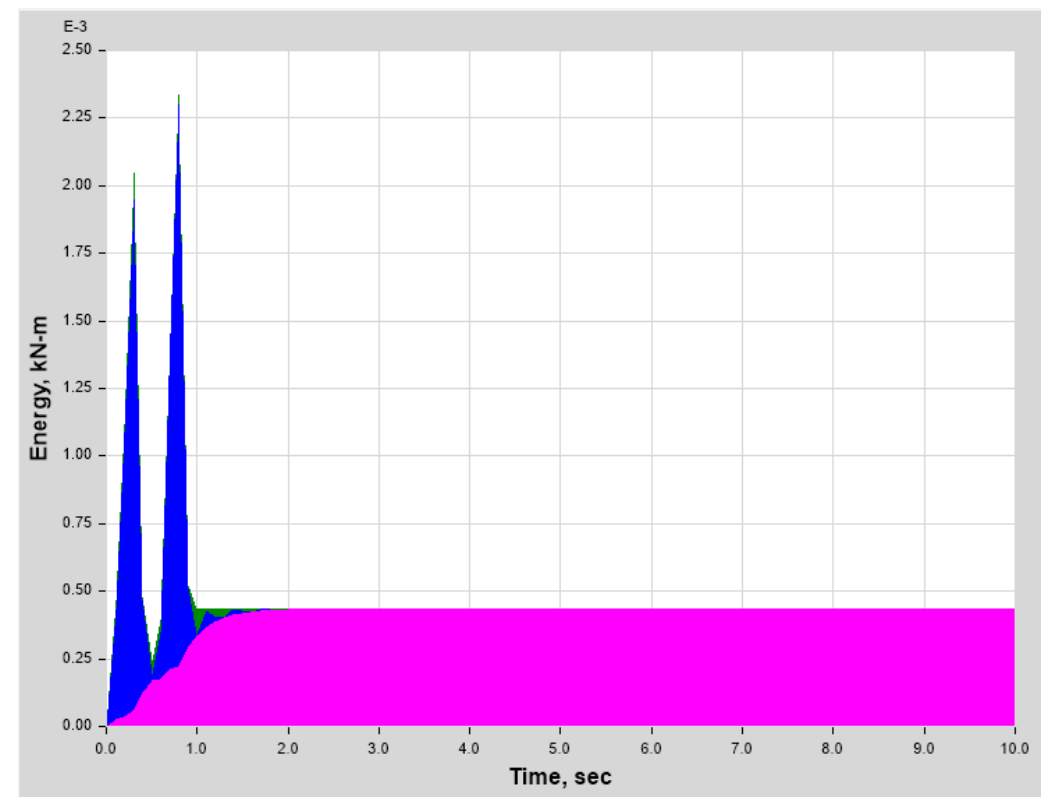

Figure 8. Cumulative Energy Components

As blast load $(18 \mathrm{KN})$ is applied on the structure, the structure absorbs $2.30 \mathrm{KN}-\mathrm{m}$ energy and vibrates for 2.5 seconds. After that the structure is deformed by releasing the energy. So the internal building is becoming safe after the blast.

After performing Non-Linear Dynamic Analysis on the trussed structure; Response Spectrum Curve (Fig. 6), Base Shear Curve (Fig. 7) and Cumulative Energy Components (Fig. 8) are created as output results by the ETABS 2015. 


\section{Conclusions}

The explosion near an important building can cause catastrophic damage to the building, formation of fragments, destruction of life-support system (air conditioning, sprinkler). Injuries and deaths can be caused by exposure to blast wave front and the building may be collapsed. Secondary effects of the explosion can hinder the evacuation of people from the building causing additional injuries and deaths. For that reason the Blast Resistant Structure is designed as a cover of the building, to protect the building from the effects of explosion. In a computerized environment for the analysis of such structural responses under blast load, special-purpose software system ETABS 2015 produces better quality results and also capable of handling a variety of dynamic loads. Accordingly, the analysis of the structure under blast load should be carefully modelled when using computerized approach to evaluate structural responses.

The string like nonlinear large vibration in steel hollow members has been observed that is absorbing the blast energy and releasing through work done by the steel members.

\section{Conflict of interest}

The authors declare no conflict of interest.

\section{References}

[1] International Research Journal of Engineering and Technology (IRJET): www.irjet.net

[2] Shifferaw, Abdulaziz. Quality of computerized blast load simulation for non-linear dynamic response analysis of framed structures. Journal of EEA. 2015; 33: 63-74.

[3] Hrvoje, Vladimir. Blast loading on structures. Technical Gazette. 19, 2012; 3: 643-652.

[4] Technical brochure of TATA STEEL - Structura Hollow Sections.

[5] Geethu, Kulbhushan, Sithara. Analysis and Design of Blast Resistant Structures. Journal of Applied Mechanics and Materials. 2017; 857: 1-6.

[6] Laurence Clough, M Eng (Hons). Synergistic Response of Steel Structures to Thermal and Blast Loading.

[7] Designers' Manual version-II by TATA STEEL - Structura Hollow Sections. 